\title{
Broadband operation of active terahertz quarter-wave plate achieved with vanadium-dioxide-based metasurface switchable by current injection
}

\author{
Toshihiro Nakanishi, ${ }^{1}$ Yosuke Nakata, ${ }^{2}$ Yoshiro Urade, ${ }^{3}$ and Kunio Okimura ${ }^{4}$ \\ ${ }^{1)}$ Department of Electronic Science and Engineering, Kyoto University, Kyoto 615-8510, \\ Japan a) \\ ${ }^{2)}$ Graduate School of Engineering Science, Osaka University, Osaka 560-8531, Japan \\ 3) Center for Emergent Matter Science, RIKEN, Saitama 351-0198, Japan \\ 4) School of Engineering, Tokai University, Kanagawa 259-1292, Japan
}

(Dated: 3 September 2020)

We demonstrate the broadband operation of a switchable terahertz quarter-wave plate achieved with an active metasurface employing vanadium dioxide. For this purpose, we utilize anisotropically deformed checkerboard structures, which present broadband characteristics compatible with deep modulation. Moreover, the metasurface is integrated with a current injection circuit to achieve state switching; this injection circuit can also be employed to monitor the electric state of vanadium dioxide. We estimate the Stokes parameters derived from the experimental transmission spectra of the fabricated metasurface and confirm the helicity switching of circularly polarized waves near a designed frequency of $0.66 \mathrm{THz}$. The relative bandwidth is evaluated as 0.52 , which is 4.2 times broader than that in a previous study.

Terahertz waves have unique properties, such as high transparency to optically opaque materials, and distinct spectral responses to molecules; they have been extensively applied in nondestructive imaging, biomaterial detection, and other $\operatorname{areas} \frac{1,2}{2}$. In the development of terahertz technologies, it is quite important to manipulate polarization, which is one of the most fundamental characteristics of electromagnetic waves. In optical regions, a birefringent material is used to realize polarization devices, such as a half- or quarter-wave plate; however, the available terahertz components for polarization control are still limited and inefficient. Furthermore, active control of terahertz polarization is much more difficult.

Recently, artificial materials composed of designed subwavelength structures, called as metamaterials, have gained significant interest for the manipulation of electromagnetic waves ${ }^{3}$. Numerous investigations have focused on two-dimensional subwavelength structures, called as metasurfaces ${ }^{4}$, which induce discontinuous phase shifts in transmitted or reflected electromagnetic waves 5.6 . Generally, anisotropic and chiral metasurfaces can modify the polarization of electromagnetic waves, owing to the phase difference between orthogonal polarizations, and various types of metasurfaces have been proposed to provide a birefringent response $\mathrm{e}^{7}$ or optical activity ${ }^{8}$. In addition, active polarization control can be realized by reconfigurable metasurfaces incorporating dynamic elements, such as microelectromechanical systems (MEMS) ${ }^{-12}$, semiconductors ${ }^{13}$, and graphene ${ }^{14}$. Phase-change materials, such as vanadium dioxide $\left(\mathrm{VO}_{2}\right)$ (which undergoes an insulator-to-metal transition near $65^{\circ} \mathrm{C}$ ), have also been utilized to achieve active polarization control ${ }^{15-18}$. Previously, we have also proposed a $\mathrm{VO}_{2}$ integrated metasurface with dipole-embedded checkerboard structures functioning as an active quarter-wave plate, whose fast and slow axes can be interchanged by increasing the tem-

\footnotetext{
${ }^{a)}$ Electronic mail: t-naka@ kuee.kyoto-u.ac.jp
}

perature using an external heater ${ }^{19}$. This metasurface can reverse the rotational direction of circularly polarized waves that are generated from linearly polarized incident waves. Generally, the broadband operation of metasurfaces is challenging, because they frequently employ resonances to induce effective electromagnetic responses. The above-mentioned active terahertz quarter-wave plate also suffers from a severely limited bandwidth, owing to its complex spectral response unique to Fano resonances 20,21 . This is resulting from the interference between the broad resonance of the checkerboard structures and the sharp resonance of the dipole structures that are embedded in the checkerboard structures to induce an anisotropic response.

In this study, we significantly broaden the operation bandwidth of a metasurface functioning as an active quarterwave plate. For this purpose, we take advantage of the broadband responses inherent to checkerboard structures 22,23 , without the use of dipole structures, which adversely affect these responses. Instead of introducing dipole structures, we anisotropically deform the checkerboard structures and achieve broadband operation of the active quarter-wave plate. In conjunction with bandwidth broadening, we integrate a current injection circuit with the metasurface, to induce a phase transition. The state of the metasurface can be controlled by injecting an electric current into the $\mathrm{VO}_{2}$ sheets incorporated in it, and the electric state of $\mathrm{VO}_{2}$ is identified by monitoring the injecting current and the applied voltage. This integrated design of a metasurface without an external heater is suitable for the miniaturization of a device.

We briefly review the design rules based on Babinet's principle $^{19}$ using an actual metasurface, whose top view is shown in Fig. 11a). The metasurface is composed of metallic sheets and variable resistive sheets whose sheet impedance $Z$ can vary over a wide range. Figures 1 b) and (c) illustrate the states, labeled as off and on states, in the limit of $Z \rightarrow \infty$ and $Z \rightarrow 0$, respectively. These two states are complementary to each other, for the inversion of the metallic and vacant 
(a) variable resistive sheet
(sheet impedance $Z$ )

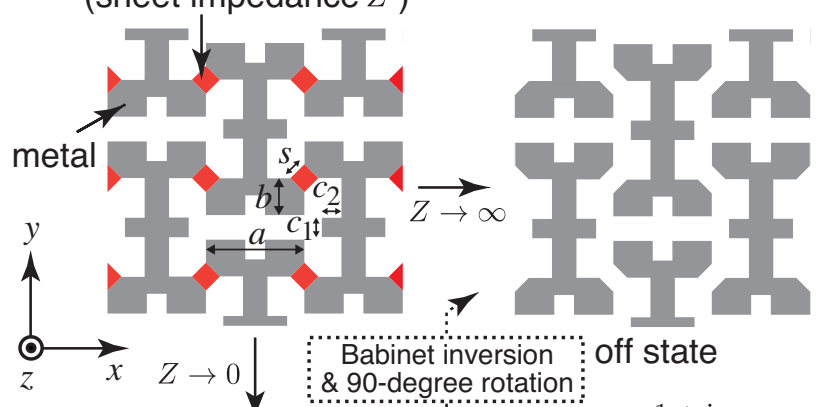

(c)
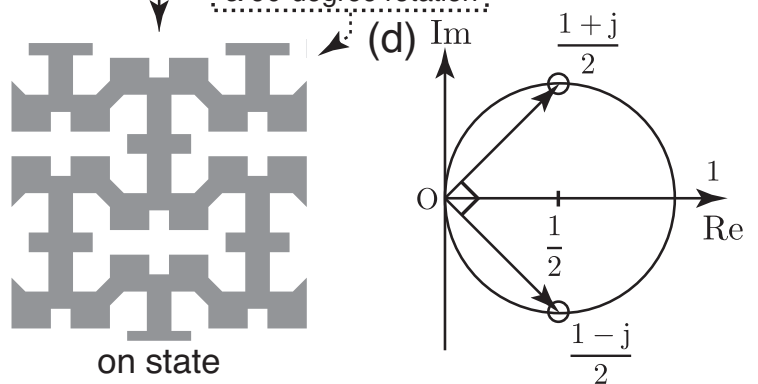

FIG. 1. (a) Top view of schematic design of metasurface. (b) Off state $(Z \rightarrow \infty)$. (c) On state $(Z \rightarrow 0)$. (d) Amplitude transmission coefficients plotted on a complex plane for realizing quarter-wave plate.

parts. The on state can be obtained by rotating the inverted structure of the off state by 90 degrees, and vice versa. From Babinet's principle applying to the metasurface with the distinct symmetry, the following relations can be derived for the complex amplitude transmission coefficients:

$$
\tilde{t}_{x}^{\text {(off) }}+\tilde{t}_{x}^{\text {(on) }}=1, \quad \tilde{t}_{y}^{\text {(off) }}+\tilde{t}_{y}^{\text {(on })}=1,
$$

where subscripts $x$ and $y$ represent the corresponding polarizations of the incident waves, and superscripts (on) and (off) represent the states of the variable resistive sheets ${ }^{24.25}$. If the single-layer metasurface with a subwavelength thickness does not have any loss, including the Ohmic dissipation and energy leakage resulting from diffraction and polarization conversion between the $x$ and $y$ polarizations, the transmission coefficients should be located on the circumference of a unit circle with the center at $1 / 2$ in a complex plane, as depicted in Fig. 1(d). Assuming that the metasurface in the off state is designed to act as a quarter-wave plate, which demands $\tilde{t}_{x}^{\text {(off) }}= \pm \mathrm{j} \tilde{t}_{y}^{\text {(off) }}$, possible solutions are provided as $\tilde{t}_{x}^{\text {(off) }}=(1 \pm \mathrm{j}) / 2$ and $\tilde{t}_{y}^{\text {(off) }}=(1 \mp \mathrm{j}) / 2$, respectively. They are shown as open circles in Fig.1(d). In this case, the magnitudes of the transmission coefficients should satisfy the following conditions 19 :

$$
\left|\tilde{t}_{x}^{\text {(off) }}(\omega)\right|=\left|\tilde{t}_{y}^{\text {(off) }}(\omega)\right|, \quad \frac{\mathrm{d}\left|\tilde{t}_{x}^{\text {(off) }}\right|}{\mathrm{d} \omega} \cdot \frac{\mathrm{d}\left|\tilde{t}_{y}^{\text {(off })}\right|}{\mathrm{d} \omega}<0
$$

where $\omega$ is an angular frequency. Babinet's relations, as expressed in Eq. (1), ensure that the on state also functions (a)

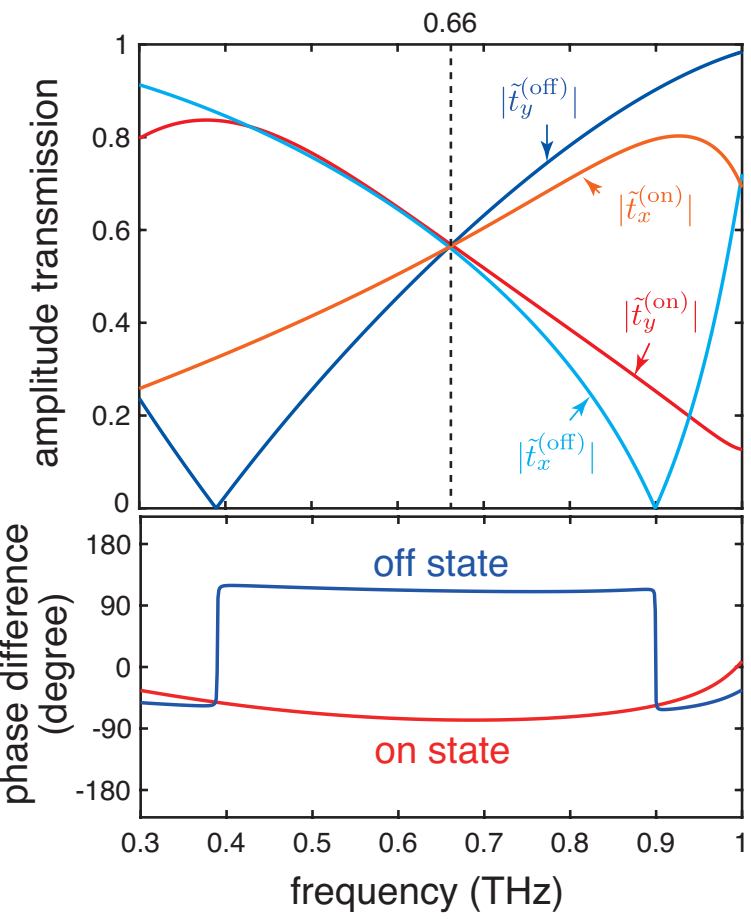

FIG. 2. (a) Normalized amplitude transmission spectra and (b) phase differences between $y$ and $x$ polarization components derived from simulation results.

as a quarter-wave plate with $\tilde{t}_{x}^{\text {(on })}=(1 \mp \mathrm{j}) / 2\left(=\tilde{t}_{y}^{(\text {off })}\right)$ and $\tilde{t}_{y}^{(\text {on })}=(1 \pm \mathrm{j}) / 2\left(=\tilde{t}_{x}^{\text {(off })}\right)$, where the slow and fast axes are interchanged compared to those for the off state.

Based on the above strategy, we design a metasurface by adjusting the dimensions of the structures as shown in Fig. 11a), using a commercial software package (CST Microwave Studio). We suppose that the metallic sheets are composed of perfect electric conductors with zero thickness and that the variable resistive sheets are in an insulating state with $Z=100 \mathrm{k} \Omega$, which is a typical sheet impedance of a 200 -nm-thick $\mathrm{VO}_{2}$ film at room temperature $\sim 25^{\circ} \mathrm{C}$ (see in Supplementary Material). The metasurface is formed on a $c$-cut sapphire substrate with semi-infinite thickness and anisotropic refractive indices of $n_{x}=n_{y}=3.1$ in the $x-y$ plane and $n_{z}=3.4$ in the propagation direction 26 . We fixed $s$ and $a$ as $s=10 \mu \mathrm{m}$ and $a=60 \mu \mathrm{m}$, respectively, which determine diffraction frequency $f=c_{0} /\left(\sqrt{2} n_{z} a\right)$ near $1.04 \mathrm{THz}$. Amplitude transmission spectra, which are normalized by that obtained for the substrate without the metasurface, for normally incident terahertz waves are calculated for the periodic system in the $x$ and $y$ directions under the periodic boundary conditions. From the simulation results, we determine the design parameters as $b=25 \mu \mathrm{m}, c_{1}=14 \mu \mathrm{m}$, and $c_{2}=15 \mu \mathrm{m}$, such that Eq. (2) for the off state is satisfied. The details of the optimization procedure are described in the Supplementary Material. Figure 2 (a) presents the calculated transmission spectra. The sheet impedance of the resistive sheets for the on state is set as $Z=10 \Omega$. As expected, for the on state, two transmission 
spectra cross at the intersection point for the off state near $0.66 \mathrm{THz}$, and Eq. (2) is also automatically satisfied for the on state. Figure 2 (b) shows the phase difference between the $y$ and $x$ polarization components, defined as $\arg \left(\tilde{t}_{y} / \tilde{t}_{x}\right)$, for both the states. The practically flat response found in the broad spectral range from $0.4 \mathrm{THz}$ to $0.9 \mathrm{THz}$ is a unique property of this metasurface. The phase differences at $0.66 \mathrm{THz}$ are estimated as $+111^{\circ}$ and $-78^{\circ}$ for the off and on states, respectively, and they slightly deviate from the ideal values of $\pm 90^{\circ}$, respectively. This is because Eqs. (11) and (2) are not strictly satisfied, mainly because the substrate breaks the reflection symmetry required for Babinet's principle. Nevertheless, the metasurface presents excellent performance as a linear-to-circular polarization converter, which will be discussed below using experimental results.

In our experimental demonstration, variable resistive sheets are composed of $\mathrm{VO}_{2}$, which exhibits insulator-to-metal transitions above the critical temperature of approximately $65^{\circ} \mathrm{C}$. Figure 3 (a) shows a photomicrograph of the metasurface fabricated on a $c$-cut sapphire substrate. The thicknesses of the $\mathrm{VO}_{2}$ and aluminum films are estimated as approximately $200 \mathrm{~nm}$ and $400 \mathrm{~nm}$, respectively. The whole structure is presented in Fig. 3 b). The $\mathrm{VO}_{2}$ patterns are formed by wet etching for the $\mathrm{VO}_{2}$ film deposited by reactive magnetron sputtering, and metallic patterns are formed by lift-off process ${ }^{23}$. The details of the fabrication procedure are provided in the Supplementary Material. A metasurface with a size of $12 \mathrm{~mm} \times 9 \mathrm{~mm}$ is fabricated at the center of the substrate. For monitoring and controlling the electric state of the $\mathrm{VO}_{2}$ films, two electrodes are introduced at the top and bottom ends of the metasurface, to inject electric currents into the $\mathrm{VO}_{2}$ patches. The electric currents are applied through electric wires, which are connected to each electrode with a conductive adhesive. Both left and right sides of the metasurface are covered by $24 \mathrm{~nm}$ thick-titanium films with a width of $1.5 \mathrm{~mm}$. The role of the titanium films is discussed subsequently.

Before terahertz measurements, the current-voltage (I-V) characteristics between the electrodes are evaluated using a direct-current power source operated in a constant-current mode. The results observed at room temperature around $24{ }^{\circ} \mathrm{C}$ with increasing current at a rate of $0.5 \mathrm{~mA} / \mathrm{s}$ are shown as a solid line in Fig. 3 (c). The dashed line represents the I$\mathrm{V}$ characteristics when the mount holding the metasurface is heated at $85^{\circ} \mathrm{C}$. The resistance characteristics $R=V / I$ derived from the $\mathrm{I}-\mathrm{V}$ characteristics are shown in Fig. 3(d). For small current $I<20 \mathrm{~mA}$, the voltage increases almost linearly with increasing current. In this region, most of the electric current is concentrated on the titanium films at the sides of the metasurface, and the resistance $R \sim 4 \mathrm{k} \Omega$ can be regarded as the resistance of the titanium films. This is because the sheet impedance of the $\mathrm{VO}_{2}$ films is extremely high $(\sim 67 \mathrm{k} \Omega)$ at room temperature. The Joule heat in the titanium sheet increases the temperature of the sapphire substrate with a high heat conductance and facilitates the phase transitions of the $\mathrm{VO}_{2}$ films. At approximately $I=45 \mathrm{~mA}$, the voltage across the electrodes abruptly drops from $V=90 \mathrm{~V}$, which suggests that some of the $\mathrm{VO}_{2}$ patches undergo a phase transition, and conducting paths in the metasurface are formed. The (a)

(b)
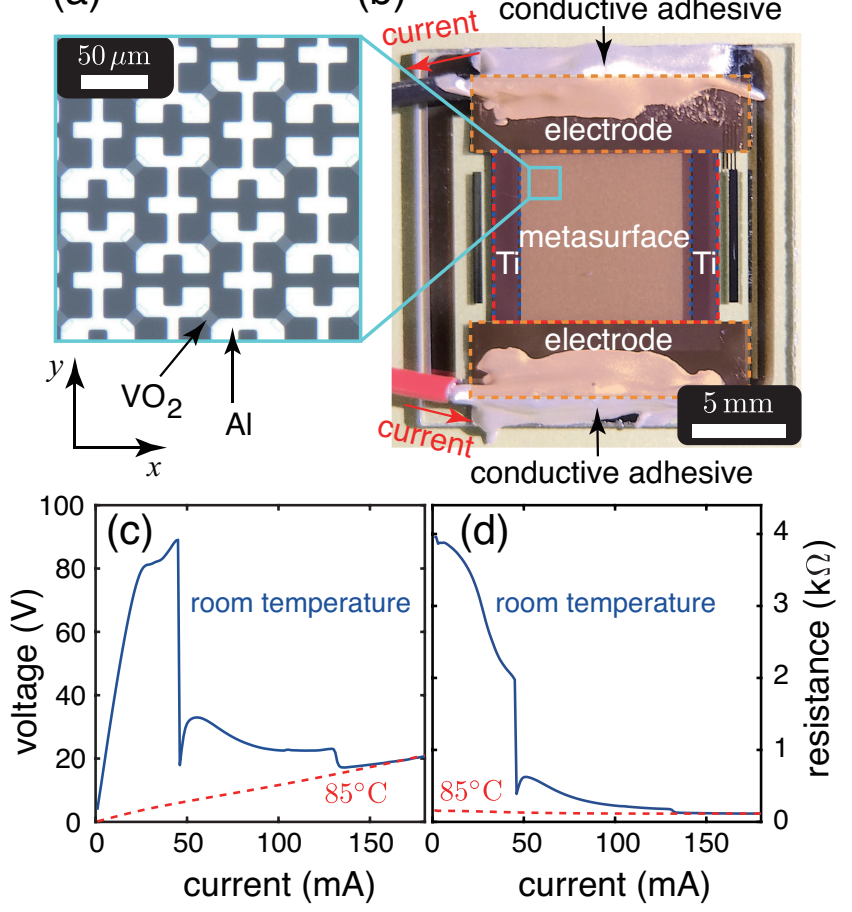

FIG. 3. (a) Photomicrograph of metasurface. (b) Whole structure of device. (c) Current-voltage characteristics between electrodes. (d) Current-resistance characteristics between electrodes.

required voltage for the phase transition would be considerably higher without the titanium sheets, which effectively reduce the threshold voltage. With increasing $I$, a ratio of $\mathrm{VO}_{2}$ patches in the metallic state is gradually increased. The I-V characteristics present a small drop close to $I=130 \mathrm{~mA}$ and asymptotically approach those of the dashed line. This suggests that the $\mathrm{VO}_{2}$ patches are completely in the metallic state for $I>130 \mathrm{~mA}$, because the dashed line represents the I-V curve at a temperature considerably higher than the critical temperature. The resistance approaches to a constant value around $120 \Omega$, which is much smaller than the resistance of the titanium films $\sim 4 \mathrm{k} \Omega$, and most of the current is concentrated on the metasurface without the titanium films. The twostep transition in Fig. 3 (c) is also observed for the conduction of a single $\mathrm{VO}_{2}$ gap, owing to the percolation processes associated with the metallic and insulating phases coexisting in a metastable state 27 . The state at $I=180 \mathrm{~mA}(V=21 \mathrm{~V})$, which corresponds to a power consumption of $3.8 \mathrm{~W}$, was used as the on state in the following experiments. The power consumption in the titanium films is estimated to be $0.11 \mathrm{~W}$, which is three percent of the total power consumption.

Subsequently, we evaluated the transmission characteristics of the metasurface via conventional terahertz time-domain spectroscopy 28 , in which complex transmission coefficients $\tilde{t}_{x}^{\text {(off) }}(\omega), \tilde{t}_{y}^{\text {(off) }}(\omega), \tilde{t}_{x}^{\text {(on) }}(\omega)$, and $\tilde{t}_{y}^{\text {(on })}(\omega)$ are derived by the Fourier transformation of the obtained signals in the time domain. The amplitude transmission is normalized by the reference signals, which are obtained using the sapphire sub- 
(a)

(b)

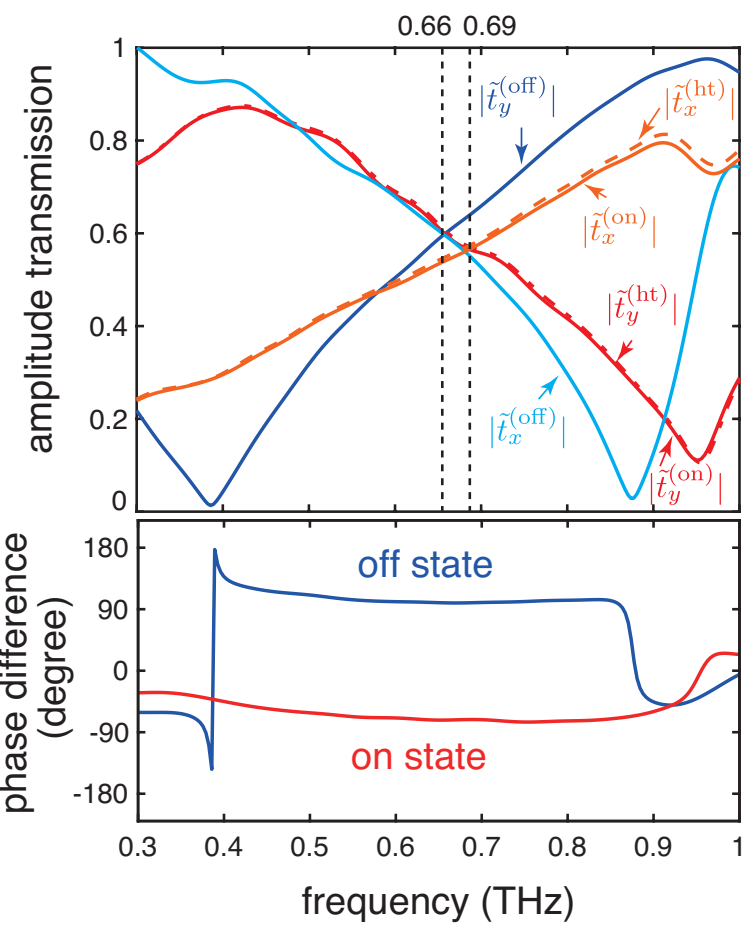

(c)

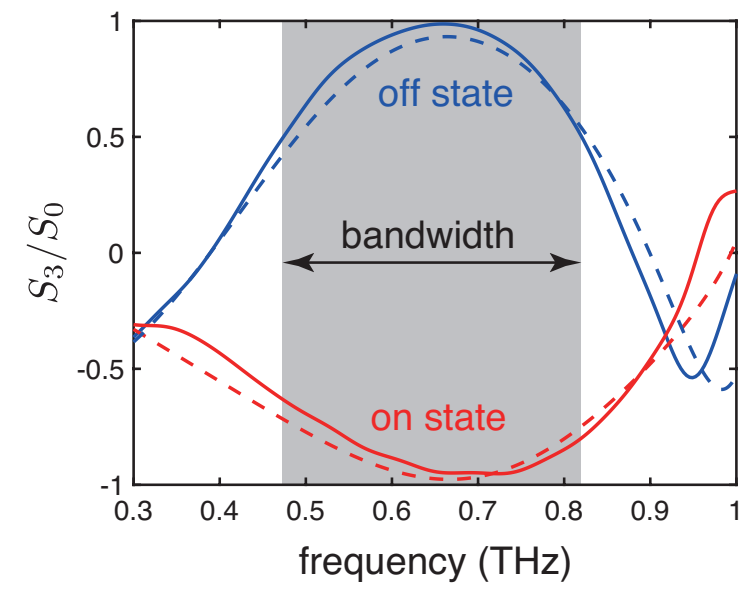

FIG. 4. (a) Solid lines represent amplitude transmission spectra for off state at $I=0$ and on state at $I=180 \mathrm{~mA}$. Dashed line labeled as $\tilde{t}_{x}^{(\mathrm{ht})}$ and dash-dotted line labeled as $\tilde{t}_{y}^{(\mathrm{ht})}$ represent the spectra at $85^{\circ} \mathrm{C}$ for $x$ and $y$ polarizations, respectively. (b) Phase differences between $y$ and $x$ polarization components derived from experimental results for off state at $I=0$ and on state at $I=180 \mathrm{~mA}$. (c) Normalized Stokes parameter $S_{3} / S_{0}$. Solid and dashed lines correspond to experimental and simulation results, respectively. Gray-shaded area corresponds to operation bandwidth satisfying $\left|S_{3} / S_{0}\right|>0.5$ for both states.

strate without the metasurface. The derived magnitudes of the transmission coefficients are shown as solid lines in Fig.4(a), where $\tilde{t}_{x}^{\text {(off) }}$ and $\tilde{t}_{y}^{\text {(off) }}$ are obtained without current injection and $\tilde{t}_{x}^{(\text {on })}$ and $\tilde{t}_{y}^{\text {(on) }}$ are obtained at $I=180 \mathrm{~mA}$. The dashed and dash-dotted lines represent transmission spectra $\tilde{t}_{x}^{(\mathrm{ht})}$ and $\tilde{t}_{y}^{(\mathrm{ht})}$ for the $x$ and $y$ polarizations, respectively, when the holder of the substrate is heated at $85^{\circ} \mathrm{C}$ without current injection into the metasurface. For both the polarization states, the obtained results at $I=180 \mathrm{~mA}$ are almost the same as those for $85^{\circ} \mathrm{C}$, and it is evident that the current injection at $I=180 \mathrm{~mA}$ is sufficient to induce a complete phase transition in the $\mathrm{VO}_{2}$ patches. The four transmission spectra, $\left|\tilde{t}_{x}^{\text {(off })}\right|,\left|\tilde{t}_{y}^{(\text {off })}\right|,\left|\tilde{t}_{x}^{(\text {on })}\right|$, and $\left|\tilde{t}_{y}{ }^{\text {on })}\right|$, obtained in the experiment agree well with the simulation results shown in Fig. 2. a). Some discrepancy possibly arises from the experimental limitations, such as wavefront deformation of the incident terahertz waves, fabrication error of the metasurface, and finite conductivity of the aluminum films. Figure $4(\mathrm{~b})$ presents the phase differences between the $y$ and $x$ polarization components for both the states. At $0.66 \mathrm{THz}$ where $\left|\tilde{t}_{x}^{\text {(off) }}\right|=\left|\tilde{t}_{y}^{\text {(off) }}\right|=0.60$ and at $0.69 \mathrm{THz}$ where $\left|\tilde{t}_{x}^{\text {(on })}\right|=\left|\tilde{t}_{y}^{(\text {on })}\right|=0.57$, the phase differences are estimated as $+99^{\circ}$ and $-71^{\circ}$ for the off and on states, respectively. The absolute power transmissions, including the Fresnel reflection loss of $-46 \%$ at both sides of the substrate, are estimated as $20 \%$ and $18 \%$, respectively.

To evaluate the function of the metasurface as an active quarter-wave plate, we use $S_{3} / S_{0}=2 \operatorname{Im}\left(\tilde{t}_{x}^{*} \tilde{r}_{y}\right) /\left(\left|\tilde{t}_{x}\right|^{2}+\left|\tilde{t}_{y}\right|^{2}\right)$, which provides one of the normalized Stokes parameters for the incidence of a 45 -degree linear polarization 29 . When the output wave is perfectly circularly polarized, $S_{3} / S_{0}$ becomes \pm 1 , whose sign corresponds to the helicity of the waves. Figure 4(c) presents the derived Stokes parameters for the off and on states. The solid and dashed lines correspond to the experimental and simulation results, respectively. It is confirmed that the helicity of the output terahertz wave is reversed from +0.99 to -0.95 close to $f_{0}=0.66 \mathrm{THz}$, where the difference in $S_{3} / S_{0}$ is maximized, and the fabricated metasurface acts as an active quarter-wave plate, as expected. Defining the operation bandwidth, $\Delta f$, as a spectral region satisfying $\left|S_{3} / S_{0}\right|>0.5$ for both the states, represented by the gray-shaded area in Fig. 4(c), we estimated $\Delta f=0.35 \mathrm{THz}$ and the relative bandwidth, $\Delta f / f_{0}=0.52$. The present study achieves a 4.2 times broader bandwidth compared to a previous result, $\Delta f / f_{0}=0.12$, with a dipole-nested checkerboard metasurface, which presents complex spectral shapes $\frac{19}{}$. Because the metasurface in this study is topologically equivalent to a simple checkerboard structure with a broad resonance, we can achieve a flat phase response, as shown in Fig. 4(b), which results in the broadband operation as an active quarter-wave plate.

In this study, we have demonstrated the broadband operation of a metasurface functioning as an active quarter-wave plate, whose fast and slow axes can be interchanged. Both the simulation and experimental results confirm that the metasurface presents excellent performance as an active quarter-wave plate, and the available bandwidth is 4.2 times broader than that in a previous study. The states of the metasurface are controlled by directly injecting electric currents, which can also be utilized to monitor the electric states of vanadium dioxide. Compared with other related studies of electrically controllable metasurfaces with $\mathrm{VO}_{2}$ films, which are connected in parallel with metallic elements $\frac{18,30-33}{3}$, the $\mathrm{VO}_{2}$ films of this metasurface are connected in series in the current direction. 
To substantially reduce the critical voltage for the series structures, a supplementary heater formed of titanium sheets is also integrated in the metasurface. This method can be applicable to various types of active metasurfaces employing vanadium dioxide. The response time is estimated to be $60-90$ seconds from the transient measurement of $\mathrm{I}-\mathrm{V}$ characteristics for sudden current change. Some studies have shown that W-doped $\mathrm{VO}_{2}$ films have lower critical temperature ${ }^{34,35}$, which could reduce the transition time. Photoinduced phase transition by ultrafast optical pulses 36,37 might be the most effective way, which could reduce the transition time to picosecond order. The broadband active quarter-wave plate enables the polarization switching of short terahertz pulses with a broad spectrum, which opens a new route for sensitive detection of chiral molecules and terahertz data transmission.

See the Supplementary Material for the electric property of $\mathrm{VO}_{2}$ film, the optimization of the design parameters, and the fabrication procedures.

The metasurface was fabricated with the help of Kyoto University Nano Technology Hub, as part of the "Nanotechnology Platform Project" sponsored by the MEXT in Japan. The present research is supported by JSPS KAKENHI Grant Nos. 17K05075, 17K17777, and 20K05360, and the Shimadzu Science Foundation.

\section{DATA AVAILABILITY}

The data that support the findings of this study are available from the corresponding author on a reasonable request.

${ }^{1}$ Y.-S. Lee, Principles of Terahertz Science and Technology (Springer, Boston, 2008).

${ }^{2}$ K.-E. Peiponen, A. Zeitler, and M. Kuwata-Gonokami, Terahertz Spectroscopy and Imaging (Springer, Berlin, 2013).

${ }^{3}$ L. Solymar and E. Shamonina, Waves in Metamaterials (Oxford University Press, New York, 2009).

${ }^{4}$ H.-T. Chen, A. J. Taylor, and N. Yu, A review of metasurfaces: physics and applications, Rep. Prog. Phys. 79, 076401 (2016)

${ }^{5}$ N. Yu, P. Genevet, M. A. Kats, F. Aieta, J.-P. Tetienne, F. Capasso, and Z. Gaburro, Light propagation with phase discontinuities: Generalized laws of reflection and refraction, Science 334, 333 (2011)

${ }^{6} \mathrm{C}$. Pfeiffer and A. Grbic, Metamaterial Huygens' surfaces: Tailoring wave fronts with reflectionless sheets, Phys. Rev. Lett. 110, 197401 (2013)

${ }^{7}$ C. Pfeiffer and A. Grbic, Bianisotropic Metasurfaces for Optimal Polarization Control : Analysis and Synthesis, Phys. Rev. Appl. 2, 044011 (2014)

${ }^{8}$ A. V. Rogacheva, V. A. Fedotov, A. S. Schwanecke, and N. I. Zheludev, Giant Gyrotropy due to Electromagnetic-Field Coupling in a Bilayered Chiral Structure, Phys. Rev. Lett. 97, 177401 (2006)

${ }^{9}$ T. Kan, A. Isozaki, N. Kanda, N. Nemoto, K. Konishi, M. KuwataGonokami, K. Matsumoto, and I. Shimoyama, Spiral metamaterial for active tuning of optical activity, Appl. Phys. Lett. 102, 221906 (2013)

${ }^{10}$ M. Zhang, W. Zhang, A. Q. Liu, F. C. Li, and C. F. Lan, Tunable Polarization Conversion and Rotation based on a Reconfigurable Metasurface, Sci. Rep. 7, 12068 (2017)

${ }^{11}$ X. Zhao, J. Schalch, J. Zhang, H. R. Seren, G. Duan, R. D. Averitt, and $\mathrm{X}$. Zhang, Electromechanically tunable metasurface transmission waveplate at terahertz frequencies, Optica 5, 303 (2018)

${ }^{12}$ L. Cong, P. Pitchappa, Y. Wu, L. Ke, C. Lee, N. Singh, H. Yang, and R. Singh, Active Multifunctional Microelectromechanical System Metadevices: Applications in Polarization Control, Wavefront Deflection, and Holograms, Adv. Opt. Mater. 5, 1600716 (2017)

${ }^{13}$ S. Zhang, J. Zhou, Y.-S. Park, J. Rho, R. Singh, S. Nam, A. K. Azad, H.T. Chen, X. Yin, A. J. Taylor, and X. Zhang, Photoinduced handedness switching in terahertz chiral metamolecules, Nat. Commun. 3, 942 (2012)

${ }^{14}$ Z. Miao, Q. Wu, X. Li, Q. He, K. Ding, Z. An, Y. Zhang, and
L. Zhou, Widely Tunable Terahertz Phase Modulation with Gate-Controlled Graphene Metasurfaces, Phys. Rev. X 5, 041027 (2015)

${ }^{15}$ D. Wang, L. Zhang, Y. Gu, M. Q. Mehmood, Y. Gong, A. Srivastava, L. Jian, T. Venkatesan, C.-W. Qiu, and M. Hong, Switchable Ultrathin Quarter-wave Plate in Terahertz Using Active Phase-change Metasurface, Sci. Rep. 5, 15020 (2015)

${ }^{16}$ D. Wang, L. Zhang, Y. Gong, L. Jian, T. Venkatesan, C. W. Qiu, and M. Hong, Multiband switchable terahertz quarter-wave plates via phasechange metasurfaces, IEEE Photonics J. 8, 5500308 (2016)

${ }^{17}$ X. Liu, X. Chen, E. P. Parrott, C. Han, G. Humbert, A. Crunteanu, and E. Pickwell-MacPherson, Invited Article: An active terahertz polarization converter employing vanadium dioxide and a metal wire grating in total internal reflection geometry, APL Photonics 3, 051604 (2018)

${ }^{18}$ M. T. Nouman, J. H. Hwang, M. Faiyaz, K.-J. Lee, D.-Y. Noh, and J.-H. Jang, Vanadium dioxide based frequency tunable metasurface filters for realizing reconfigurable terahertz optical phase and polarization control, Opt. Express 26, 12922 (2018)

${ }^{19}$ Y. Nakata, K. Fukawa, T. Nakanishi, Y. Urade, K. Okimura, and F. Miyamaru, Reconfigurable terahertz quarter-wave plate for helicity switching based on Babinet inversion of anisotropic checkerboard metasurface, Phys. Rev. Appl. 11, 044008 (2019)

${ }^{20}$ A. E. Miroshnichenko, S. Flach, and Y. S. Kivshar, Fano resonances in nanoscale structures, Rev. Mod. Phys. 82, 2257 (2010)

${ }^{21}$ E. Kamenetskii, A. Sadreev, and A. Miroshnichenko, Fano Resonances in Optics and Microwaves (Springer, Cham, 2018).

${ }^{22}$ K. Takano, F. Miyamaru, K. Akiyama, H. Miyazaki, M. W. Takeda, Y. Abe, Y. Tokuda, H. Ito, and M. Hangyo, Crossover from capacitive to inductive electromagnetic responses in near self-complementary metallic checkerboard patterns, Opt. Express 22, 24787 (2014)

${ }^{23}$ Y. Urade, Y. Nakata, K. Okimura, T. Nakanishi, F. Miyamaru, M. W. Takeda, and M. Kitano, Dynamically Babinet-invertible metasurface: a capacitive-inductive reconfigurable filter for terahertz waves using vanadium-dioxide metal-insulator transition, Opt. Express 24, 4405 (2016)

${ }^{24}$ Y. Nakata, Y. Urade, T. Nakanishi, and M. Kitano, Plane-wave scattering by self-complementary metasurfaces in terms of electromagnetic duality and Babinet's principle, Phys. Rev. B 88, 205138 (2013)

${ }^{25}$ Y. Nakata, Y. Urade, K. Okimura, T. Nakanishi, F. Miyamaru, M. W. Takeda, and M. Kitano, Anisotropic Babinet-Invertible Metasurfaces to Realize Transmission-Reflection Switching for Orthogonal Polarizations of Light, Phys. Rev. Appl. 6, 044022 (2016)

${ }^{26}$ D. Grischkowsky, S. Keiding, M. van Exter, and C. Fattinger, Far-infrared time-domain spectroscopy with terahertz beams of dielectrics and semiconductors, J. Opt. Soc. Am. B 7, 2006 (1990)

${ }^{27}$ Y. Zhao, J. Hao, C. Chen, and Z. Fan, Electrically controlled metal-insulator transition process in $\mathrm{VO}_{2}$ thin films, J. Phys. Condens. Matter 24, 035601 (2012)

${ }^{28}$ M. Hangyo, M. Tani, and T. Nagashima, Terahertz Time-Domain Spectroscopy of Solids: A Review, Int. J. Infrared Millimeter Waves 26, 1661 (2005)

${ }^{29}$ B. E. A. Saleh and M. C. Teich, Fundamentals of photonics; 2nd ed. (Wiley, New York, 2007).

${ }^{30}$ Z. Zhu, P. G. Evans, R. F. Haglund, and J. G. Valentine, Dynamically Reconfigurable Metadevice Employing Nanostructured Phase-Change Materials, Nano Lett. 17, 4881 (2017)

${ }^{31}$ C. Han, E. P. Parrott, G. Humbert, A. Crunteanu, and E. PickwellMacPherson, Broadband modulation of terahertz waves through electrically driven hybrid bowtie antenna- $\mathrm{VO}_{2}$ devices, Sci. Rep. 7, 12725 (2017)

${ }^{32}$ G. Zhou, P. Dai, J. Wu, B. Jin, Q. Wen, G. Zhu, Z. Shen, C. Zhang, L. Kang, W. Xu, J. Chen, and P. Wu, Broadband and high modulationdepth $\mathrm{THz}$ modulator using low bias controlled $\mathrm{VO}_{2}$-integrated metasurface, Opt. Express 25, 17322 (2017)

${ }^{33}$ C. Zhang, G. Zhou, J. Wu, Y. Tang, Q. Wen, S. Li, J. Han, B. Jin, J. Chen, and $\mathrm{P}$. Wu, Active Control of Terahertz Waves Using Vanadium-DioxideEmbedded Metamaterials, Phys. Rev. Appl. 11, 054016 (2019)

${ }^{34} \mathrm{~T}$. Hörlin, T. Niklewski, and M. Nygren, Electrical and magnetic properties of $\mathrm{V}_{1-\mathrm{x}} \mathrm{W}_{\mathrm{x}} \mathrm{O}_{2}, 0 \leq \mathrm{x} \leq 0.060$, Mat. Res. Bull. 7, 1515 (1972).

${ }^{35}$ G. Karaoglan-Bebek, M. N. F. Hoque, M. Holtz, Z. Fan, and A. A. Bernussi, Continuous tuning of $\mathrm{W}$-doped $\mathrm{VO}_{2}$ optical properties for terahertz analog applications, Appl. Phys. Lett. 105, 201902 (2014). 
${ }^{36}$ M. Nakajima, N. Takubo, Z. Hiroi, Y. Ueda, and T. Suemoto, Photoinduced metallic state in $\mathrm{VO}_{2}$ proved by the terahertz pump-probe spectroscopy, Appl. Phys. Lett. 92, 011907 (2008).

${ }^{37}$ X. Xue, M. Jiang, G. Li, X. Lin, G. Ma, and P. Jin, Photoinduced insulatormetal phase transition and the metallic phase propagation in $\mathrm{VO}_{2}$ films investigated by time-resolved terahertz spectroscopy, J. Appl. Phys. 114, 193506 (2013). 


\section{Supplementary material: \\ Broadband operation of active terahertz quarter-wave plate achieved with vanadium-dioxide-based metasurface switchable by current injection}

\section{ELECTRIC PROPERTY OF $\mathrm{VO}_{2}$ FILM}

Figure 5 shows a typical sheet impedance of a 200-nmthick $\mathrm{VO}_{2}$ sheet, which is fabricated by the procedures given in Sec. III in the Supplementary Material, for various temperatures. This is obtained by a four-probe method, whose results can be applied for terahertz waves. The solid (dashed) line corresponds to the sheet impedance with increasing (decreasing) temperature. The typical sheet impedances of 200-nmthick $\mathrm{VO}_{2}$ sheets are $40 \mathrm{k} \Omega-150 \mathrm{k} \Omega$ at $25^{\circ} \mathrm{C}$ and $8 \Omega-12 \Omega$ at $100^{\circ} \mathrm{C}$, respectively. Hence, we assume the sheet impedances as $10 \Omega$ for the metallic state and as $100 \mathrm{k} \Omega$ for the insulating state when designing the metasurface.

\section{OPTIMIZATION OF DESIGN PARAMETERS}

Figures 6(a) and (b) represent the normalized amplitude transmission spectra $\left|\tilde{t}_{x}^{\text {(off) }}\right|$ and $\left|\tilde{t}_{y}^{\text {(off) }}\right|$, respectively, for three sets of parameters: (i) $b=15 \mu \mathrm{m}, c_{1}=c_{2}=15 \mu \mathrm{m}$ (solid lines); (ii) $b=20 \mu \mathrm{m}, c_{1}=c_{2}=15 \mu \mathrm{m}$ (dashed lines); (iii) $b=15 \mu \mathrm{m}, c_{1}=c_{2}=20 \mu \mathrm{m}$ (dash-dotted lines). The other parameters are fixed as $s=10 \mu \mathrm{m}$ and $a=60 \mu \mathrm{m}$. The transmission spectra are normalized by that without the metasurface, to exclude the Fresnel reflection at the surfaces of the sapphire substrate. For the $x$ polarization, the spectra present significant red shifts with increasing $c_{1}$ (or $c_{2}$ ), whereas they are insensitive to the change in $b$. However, for the $y$ polarization, the spectra present red shifts with increasing $b$, whereas they are almost independent of $c_{1}$ (or $c_{2}$ ). Consequently, it is possible to tailor the transmission spectra for the $x$ and $y$ po-

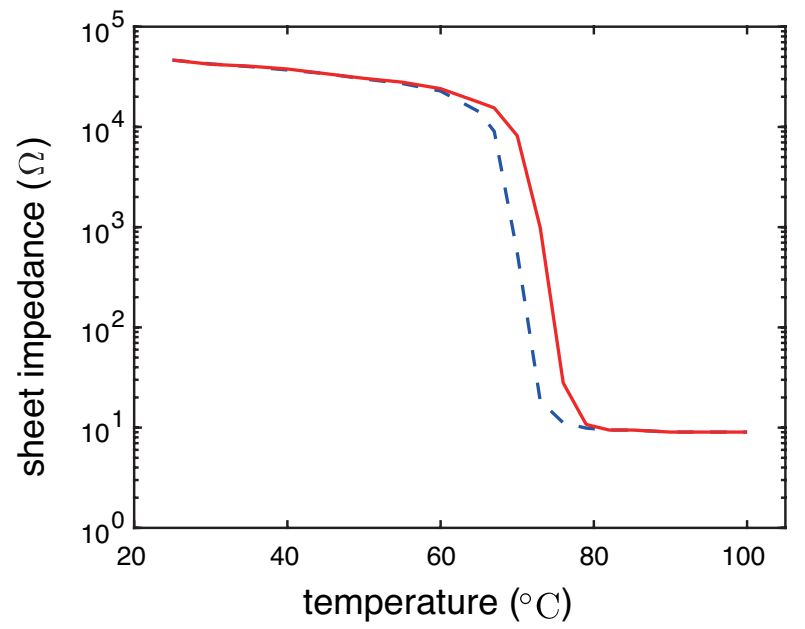

FIG. 5. Typical sheet impedance of 200-nm-thick $\mathrm{VO}_{2}$ film for various temperatures. The solid (dashed) line corresponds to the sheet impedance with increasing (decreasing) temperature. larizations almost independently, and we can easily adjust the dimensions of $b, c_{1}$, and $c_{2}$ to satisfy the conditions to realize a quarter-wave plate, as expressed in Eq. (2).

\section{FABRICATION PROCEDURES}

The metasurface is composed of three layers: a vanadium dioxide layer as a variable resistive sheet, an aluminum layer as a conductive sheet, and a titanium layer as a supplemental heater. The fabrication procedure of the metasurface is as follows. $\mathrm{A} \mathrm{VO}_{2}$ film is deposited on a $c$-cut sapphire substrate of size $20 \mathrm{~mm} \times 20 \mathrm{~mm} \times 1 \mathrm{~mm}$ by reactive magnetron sputtering with a vanadium target. The thickness of the film is estimated as approximately $200 \mathrm{~nm}$. After the $\mathrm{VO}_{2}$ pattern is formed by wet etching, metallic structures are patterned by a lift-off process using a 400-nm-thick aluminum film, which is deposited by electron-beam evaporation. Finally, a supplemental heater is fabricated by a lift-off process using a 24-nm-thick titanium film formed by electron-beam evaporation.

(a)

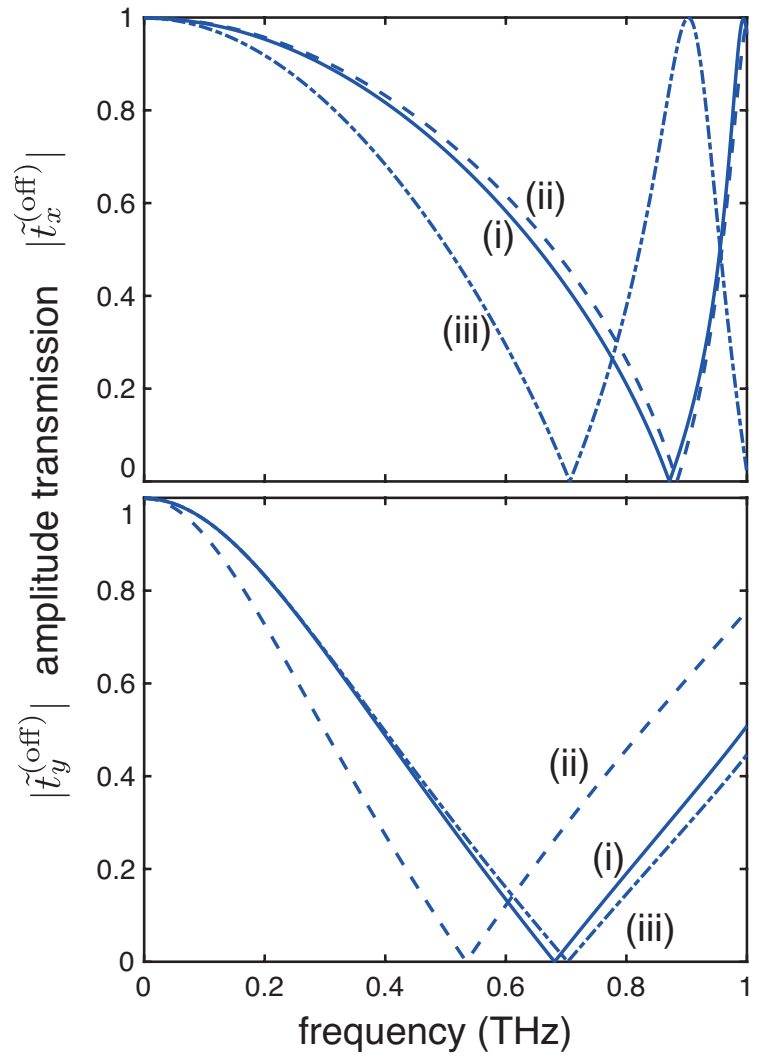

FIG. 6. Normalized transmission spectra of off state for (a) $x$ polarization and (b) $y$ polarization. Design parameters are (i) $b=15 \mu \mathrm{m}$, $c_{1}=c_{2}=15 \mu \mathrm{m}$; (ii) $b=20 \mu \mathrm{m}, c_{1}=c_{2}=15 \mu \mathrm{m}$; (iii) $b=15 \mu \mathrm{m}$, $c_{1}=c_{2}=20 \mu \mathrm{m}$. 